\title{
Clinical uses and toxicity of Ergot, Claviceps purpurea An evidence-based comprehensive retrospective review (2003-2017)
}

\author{
Raghad Al-Omari ${ }^{1}$, Atheer Al-Enazi ${ }^{1}$, Rizwan Ahmad², Niyaz Ahmad ${ }^{3}$, Atta Abbas Naqvi ${ }^{4}$ \\ and Adeeb Shehzad ${ }^{5}$ \\ ${ }^{1}$ College of Clinical Pharmacy, Imam Abdulrahman Bin Faisal University, Dammam, Saudi Arabia \\ ${ }^{2}$ Natural Products and Alternative Medicines, College of Clinical Pharmacy, Imam Abdulrahman Bin Faisal \\ University, Dammam, Saudi Arabia \\ ${ }^{3}$ Department of Pharmaceutics, College of Clinical Pharmacy, Imam Abdulrahman Bin Faisal University, \\ Dammam, Saudi Arabia \\ ${ }^{4}$ Department of Pharmacy Practice, College of Clinical Pharmacy, Imam Abdulrahman Bin Faisal University, \\ Dammam, Saudi Arabia \\ ${ }^{5}$ Department of Biomedical Engineering and Sciences, School of Mechanical and Manufacturing Engineering, \\ National University of Sciences and Technology, Islamabad, Pakistan
}

\section{ABSTRACT}

Ergot alkaloids produced by the fungus Claviceps parasitizing on cereals presents a high toxicity risk for animals and humans due to presence of its alkaloid content. Ergot-alkaloid toxicity occurs via their medicinal use however human poisoning from ergot plant is rare. The aim of this review is to determine the toxicity of ergot plant or ergotamine derivatives in humans. Databases such as Google scholar, PubMed, Scopus, Web of Science alongwith related books and theses as well as the library resources from Imam Abdulrahman Bin Faisal University were used whereas the Key words searched included; Ergot, Egotism, Ergotamine poisoning, Ergotamine interaction with HIV therapy, Ergotamine overdose, and report cases of ergotism. The relevant literature search suggests many toxicity cases and side effects associated with the use of ergot-alkaloids. More commonly it develops a condition known as ergotism i.e. a disease or toxic condition produced by eating ergot grains or rye or chronic use of ergot drug. The characteristic

\section{ARTICLE INFORMATION:}

*Corresponding Author: rizvistar_36@yahoo.com Received $1^{\text {st }}$ July, 2018

Accepted after revision $21^{\text {st }}$ Sep, 2018

BBRC Print ISSN: 0974-6455

Online ISSN: 2321-4007 CODEN: USA BBRCBA

\% Thomson Reuters ISI ESC / Clarivate Analytics USA and

Crossref Indexed Journal

NAAS Journal Score 2018: 4.31 SJIF 2017: 4.196

( $)$ A Society of Science and Nature Publication, Bhopal India 2018. All rights reserved.

Online Contents Available at: http//www.bbrc.in/

DOI: $10.21786 / \mathrm{bbrc} / 11.3 / 2$ 
features of ergotism consist of painful and cold extremities. Furthermore, a notable and severe interaction of ergotalkaloids was found in HIV patients using ergotamine and antiretroviral drugs together. Ergotamine which is one of ergot preparation has poisonous effect when taken in over dose and it interacts with antiretroviral drugs also.

KEY WORDS: EGOTISM; MIGRAINE HEADACHE; POSTPARTUM HEMORRHAGE; ARTERIAL SPASM; CYANOSIS

\section{INTRODUCTION}

The ergot fungus (Claviceps purpurea) is family of secondary metabolites and is a common seed pathogen of grasses and cereals. Ergot is a parasitic fungus that belongs to the genus Claviceps and forms dark sclerotia on various grasses and grains. After maturing, the sclerotia fall to the ground and remain partially buried for the resting stage of ergot formation. This period occurs during cold or winter weather, (Ehrlich et al., 2013). Ergot plant has medicinal uses to treat some diseases; even though, Ergot contain alkaloids which can cause severe toxicity in mammals when ingested and thus the fungal infection might provide protection for the host plant against mammalian herbivores, (Panaccione et al., 2012). Ergot alkaloids are natural products having nitrogen indole alkaloids. There are three group of ergot; I. peptides (ergopeptines), II. Clavines and III. Lysergic acid amides, (Gerhards et al., 2014).

There are more than fourty known ergot and usually six of them may cause toxicity by interaction with neurotransmitters. These are ergotamine, ergocornine, ergocryptine, ergocristine, ergosine and ergometrine, (Mulac et al., 2011). Ergot alkaloids have pharmacological and toxicological effect on several receptor systems in the human body. Ergot become activated in the body to some receptors and show cytotoxic affects and induces apoptosis in human primary cells, (Mulac et al., 2013).

Ergotism occurs when either the fungus is ingested via the contamination of grain, for example, rye or by the medicinal use of drugs derived from ergotamine compounds. Ergot toxicity called Ergotism previously known as Holy Fire, in some cases may lead to death, (Floss, 1976). There are two forms of Ergotism; gangrenous and convulsive and both can occur in the same individual. Gangrenous named as Saint Anthony's, usually characterized by pain in one limb particularly the calf and leg, inflamed, swollen, and violet hand and foot whereas in severe cases the limb will separate from the knee with hemorrhage and may need a knee amputation. The other form of ergotism i.e. convulsive form is accompanied with heaviness and numbness in limbs with paresthesia well as diarrhea without vomiting. In humans, ergot is used pharmacologically to inhibit lactation, postpartum hemorrhage, and induce labor, (Lee and Coll, 2009). Previously ergot was used in the treatment of Parkinsonism and other endocrine and neurological disorders, (Tesh, 2015).
In addition, ergot induces cytotoxic and apoptotic effect shows anti-cancer effect and thus used as a cancer therapy. In a study on two cancer lines (Hep-G2 and HT-29) ergot showed a high grade apoptotic effect, (Mulac et al., 2013). Furthermore, ergot alkaloids have an important widespread use in migraine headaches and 5-hydroxytryptamine (5-HT) receptors acts as binding site for these ergot derivatives such as Ergotamine, Dihydroergotamine, and Methysergide. They work as agonist of 5-HT1B, 5-HT1D, and 5-HT1F receptors to treat for treatment as well as prophylaxis for migraine headache with a high efficacy, (Dholf et al., 2012). Though in human, ergot complications i.e. ergotism appears rarely with symptoms such as tachycardia, hypertension and arterial spasm, still it is important to be taken care as ergot acts as a strong vasoconstrictor and may cause severe adverse effects at cardiac and limbs level, (Adam et al., 2014).

Current review aims to highlight the more important and recently reported cases regarding ergotism in humans with major symptoms observed, as associated with the use of ergot-alkaloids. The literature will be searched to extract out the factors behind ergotism and to report the possible measure in order to tackle such reasons. The study owes importance as it will compare the therapeutic and toxic profile for ergot and to conclude an overall scenario of how to use ergot-alkaloids for therapeutic purposes and to avoid ergotism.

\section{MATERIAL AND METHODS}

Various databases searched were: Google scholar, Science direct, Research gate, Web of Science, PubMed, Science Finder, Scopus and Journals such as; Journal of Ethnopharmacology, Frontier in Ethnopharmacology, Thieme journals, Clinical Toxicology journal, Journal of Agricultural and Food Chemistry, Journals of Toxicology, Canadian Medical Association Journal, JSM foot and ankle journal, Toxicology and Industrial Health (SAGE journals) and The Royal College of Physician of Edinburgh were searched. In addition, books and theses and online as well as hard resources from library of Imam Abdulrahman Bin Faisal University Damam, Saudi Arabia was also searched.

\section{Key words were also searched:}

Ergot, Ergot alkaloids, reported case of Ergot toxicity, adverse effects of ergot; ergotism, ergotamine toxicity, 
clinical cases observed for ergot alkaloids, uses of ergot alkaloids, interactions of ergot alkaloids.

The literature was searched for retrospective fifteen years i.e. 2003-2017. All the clinical cases regarding ergot toxicity or ergotism were gathered and filtered as per the inclusion and exclusion criteria as mentioned below;

\section{Inclusion criteria}

The clinical cases reported in humans associated with the use of ergot or ergot alkaloids in any of its form used, were included. The toxicity resulted due to overdose, long term use as well as any adverse effect and drug interaction of ergot and ergot derivatives with HIV drugs were also included in the study.

\section{Exclusion criteria}

Clinical cases regarding ergot toxicity, reported in animals or in vivo studies (cell lines) as well as in vitro studies were excluded from the study. Any clinical report with minor side effects or adverse effects was also excluded. Similarly, any interaction with conventional medicine other than ergotism was excluded from the study.

\section{Search result}

The number of clinical cases, after an in-depth and extensive review as well as the cases which met the inclusion/exclusion criteria was found to be ten. These cases are reported in detail in the forthcoming section of literature review;

\section{LITERATURE REVIEW}

The ten cases filtered as per eligibility criteria are explained in detail as below. These cases are reported here-in an ascending year wise order;

\section{A. Clinical case reported in 2003}

\section{i. Overdose of Ergot preparation}

A 36-years old man was diagnosed with HIV and lymphoma eight years ago and he was taking triple antiviral therapy. He developed bilateral claudication and foot cyanosis. History revealed that the patient was taking $2 \mathrm{mg}$ of ergotamine tartrate due to migraine headache. The overdose of ergot-alkaloid developed paresthesia, and coolness in feet without pain. The left foot was cyanotic and he was administered with heparin IV and oral nifedipine. The symptoms resolved within one week of the therapy, (Badwin and Ceraldi, 2003).

\section{B. Clinical case reported in 2005}

\section{ii. Ergot toxicity in neonate}

An infant born at 41 weeks gestation period was accidentally administered with methylergonovine (I.M.) instead of naloxone for respiratory depression. Soon after administration he suffered from capillary refill, low oxygen saturation, his hand and feet became warm and pink and after sometime his color became grey with development of hypercarbia (partial pressure of carbon dioxide). The infant was put on mechanical ventilation and treated with nitroprusside infusion. The condition recovered with 10 days of hospital treatment, (Bangh et al., 2005).

\section{Clinical case reported in $\mathbf{2 0 0 9}$}

\section{iii. Ergotamine with caffeine}

A 28-year old patient reported to hospital with pain in both legs. Upon history it was revealed that the patient used ergotamine tartrate $(1 \mathrm{mg})$ with caffeine (100 mg) for 3 days, due to a bi temporal headache a week ago. Thus he developed the symptoms of severe leg pains, especially below the ankle with cold and purple legs. Hands were also cold but without any pain. The patient was treated with I.V crystalline fluids and nitroprusside as well as oral nifedipine (every $8 \mathrm{~h}$ ). Papaverine was also administered as the patient had arterial spasm whereas Enoxaparine was administered in order to inhibit any thrombus formation, (Musikatavorn et al., 2009).

\section{Clinical case reported in $\mathbf{2 0 0 9}$}

\section{iv. Ergotamine with Anti-HIV}

A 31-year female patient on anti-HIV therapy used ergotamine for migraine headache. Soon after she start to complain about pain in lower extremity and her both legs were cold particularly the left leg. In addition, a palpable popliteal artery with peripheral pulses was also observed. This condition is termed as Ergotism. The sign and symptoms resolved gradually with the use of Nifedipine (30 mg) and Enoxiparine was used as treatment for Ergotism, (Cagatay et al., 2009).

\section{E. Clinical case reported in $\mathbf{2 0 1 0}$}

\section{v. Ergotamine used with clarithromycin (Antibiotic)}

An eighteen year ole female patient reported with a pain in emergency which started 2 days ago. The symptoms she presented were; cool cyanotic lower extremities, tender on palpitation, pain at rest, a thin iliac to popliteal region and blood flow with increased velocity. The history revealed that the patient was using ergotamine since 3 year for migraine whereas at the same time she used clarithromycin for upper respiratory tract infection. Heparin was administered in order to prevent possible thrombotic complications and infusion of bupivacaine was given. The symptoms resolved within a month, (Demir et al., 2010). 


\section{F. Clinical case reported in $\mathbf{2 0 1 0}$}

\section{vi. Ergotamine interaction with anti-HIV drugs produces} paresthesia

A 29-year old patient using antiretroviral therapy for HIV traveled Egypt and developed a severe migraine. A local physician prescribed him ergotamine tartrate for two weeks. Soon after its use, he developed sever pain in left upper extremities, pale and cool wooden hand with paresthesia, absent arterial pulse and axillary artery stenosis, and he was facing difficulty in writing. Heparin was started as therapy to inhibit any coagulation. Prostacyclin IV and topical Nitroglycerine was used as therapy. The symptoms resolved within six days, (Frohlich et al., 2010).

\section{G. Clinical case reported in 2012}

\section{vii. Neonate and methylergonovine}

A female neonate, 10 minutes after her delivery, was given methylergonovine instead of vitamin-K. after 30 minutes, she developed respiratory depression with cyanosis in extremities. Naloxone IM was administered to recover the symptoms, (Sullivan et al., 2012).

\section{H. Clinical cases reported in 2014}

\section{viii. Ergotamine with azithromycin (Antibiotic)}

A 35-year old women reported with severe pain and pallor in left foot, into emergency room. She was using ergotamine since long time due to migraine, and in addition she used azithromycin for four days (as she was heavy smoker). Upon examination, vasospasm and acute arterial embolism was observed due to interaction between azithromycin and ergotamine. For treatment purpose, ergotamine was stopped and Heparin was administered for a week. The symptoms resolved gradually,( Adam et al., 2014).

\section{Clinical cases reported in $\mathbf{2 0 1 6}$}

\section{ix. Ergotamine use with caffeine}

A 33-year old women presented with critical limb ischemia, foot camping pain and a decreased walking distance for 36 hours. Two days ago she used ergotamine tartrate $(1 \mathrm{mg})$ and caffeine $(100 \mathrm{mg})$ for migraine headache. She was treated with vasodilator (PG-E1 I.V.), pentoxifylline, heparin and sildenafil for every 8 hours. The patient recovered partially within 4 days, however for full recovery she was further prescribed with aspirin, sildenafil and cilostazol, (Eduardo et al., 2016). Ergotamine overdose develops cerebral ergotism

A 49-year old patient with a history of HIV infection treated with ritonavir used ergotamine (3g) for migraine. He developed cerebral ergotism with an ischemic attack after 20 minutes. The Cervical Doppler ultrasound revealed a narrowing in both internal carotid arteries. The treatment plan consist of; stopping ergotamine, and changing the antiretroviral regimen, aspirin (325 mg) was administered and bed rest suggested. The patient became asymptomatic after a week. (Gaye-Saavedra et al., 2016) The cases reported for Ergot are presented in detail in Table 1, as below;

\section{DISCUSSION}

Ergot, with the most prominent member in group i.e. Claviceps purpurea, grows on rye and other grasses. It is considered as a poisonous plant that produce ergotism in humans and sever toxicity in mammals. The improper use or overdose of ergot may lead to various severe complications, discussed as below.

Ergot develops psychological effects; convulsive, spasmodic or nervous ergotism i.e. chronic serious stage of ergotism called "convulsive stage", can occur due to patchy damage of mid brain and cerebral hemisphere which leads to degeneration in the posterior column of spinal cord. The patient in such cases suffers from opisthotonos strong muscle spasm, tonic or clonic spasm of limbs, battened tongue, dilated pupils, mania, dementia, glaucoma, and delirium. In addition, the patient may develop status epilepticus/multiple convulsions with less or lack of sleep and finally coma and death, (Lee and Coll, 2009).

Ergonovine evoke coronary artery spasm (CAS); earlier, ergonovine (i.v.) was considered as a useful test for CAD diagnosis however, recently it has been reported that it may provoke variant angina in patients. It effects vascular smooth muscles via alpha adrenergic agonist activity and leads to vasoconstriction. Similarly, in CNS it blocks the baroreceptors and prevents response of reflex bradycardia. Thus it is considered an unsafe drug in $\mathrm{CAD}$ patients because ergonovine may develop severe hypertension, myocardial infarction, seizures, intracerebral hemorrhage and death, (Curry and Pepine, 1977).

Acute coronary syndrome with myocardial bridging; Ergotamine may induce vasoconstriction in coronary, cerebral and pulmonary arteries and leads to change in ECG pattern. A case of ergotamine overdose was reported in 2003, for a 48-year aged women with migraine headache, whereby she developed recurrent chest pain with a complaint since last 10 days. The ECG showed T-wave inversion and elevated ST-segment. This shows that ergotamine may cause serious cardiac adverse effects such as; arrhythmias, coronary vasospasm and death, (Shimony et al., 2006).

Induces serotonin syndrome; it can be a mild to life-threatening syndrome. Dihydroergotamine, binds to serotonin receptors in the dorsal horn of spinal cord, result in serotonergic hyper-stimulation and may 
Raghad Al-Omari et al.

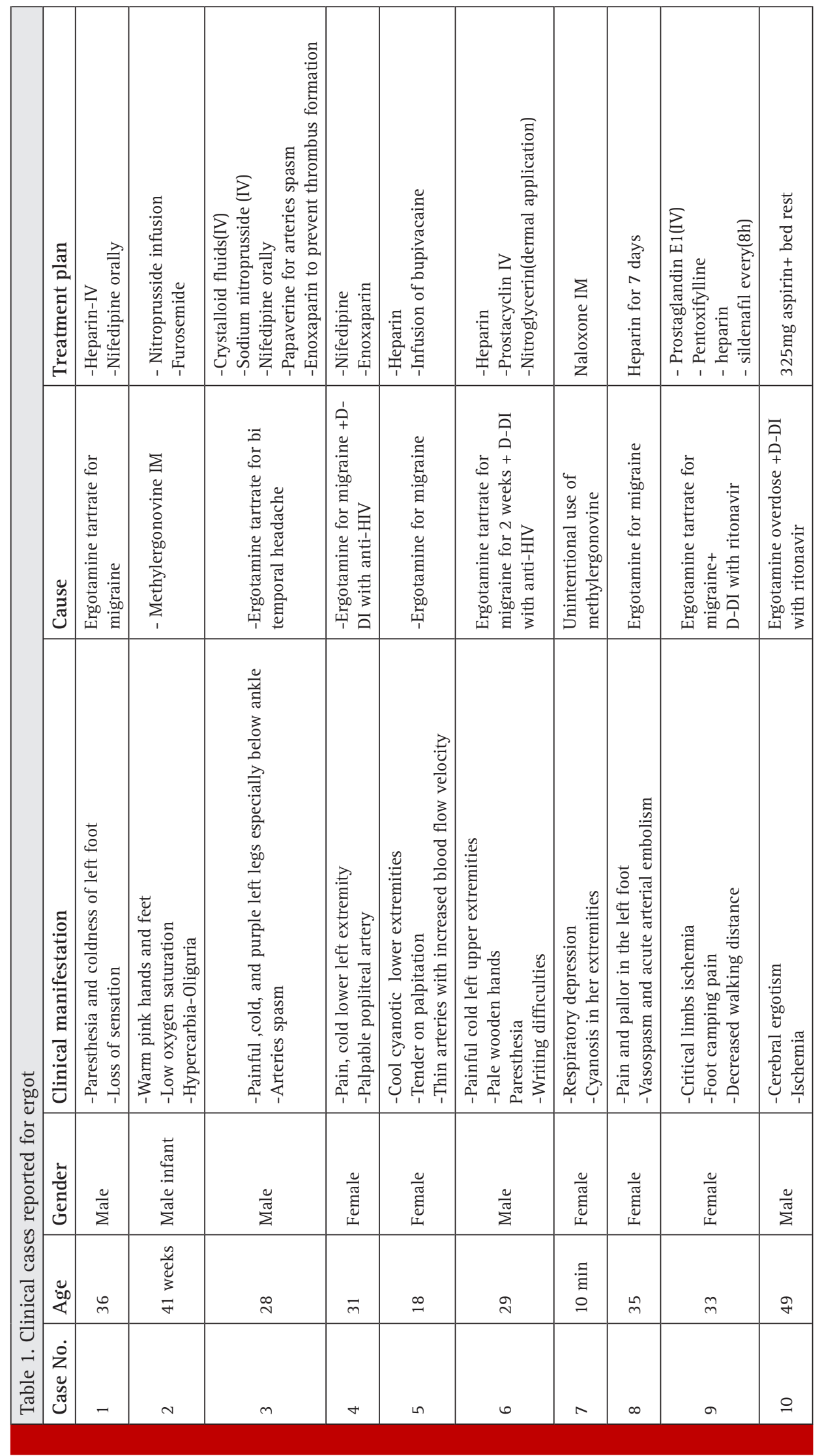

360 A COMPREHENSIVE REVIEW OF ERGOT

Bioscience Biotechnology Research Communications 
develop "convulsive ergotism" characterized with symptoms of behavioral changes and mental disturbances, agitation, sweating, fever, and hallucination etc. (Eadie, 2003).

Ergotamine and its interaction with macrolide antibiotics; the combination of ergotamine with erythromycin is considered and reported as a dangerous combination. The reason behind is; macrolides have hepatic circulation with ergotamine whereby it causes severe vascular spasm and transient renal ischemia. And this is due to macrolide inhibition of cytochrome P-450 metabolism leading to an increase serum ergotamine concentration. (Ghali et al., 1993) A clinical case reported in 2010 about a woman with sore throat and migraine. She was prescribed with erythromycin (500 mg) and ergotamine (100 $\mathrm{mg}$ ) and soon after the combined use of these drugs she developed pain and paresthesia in both feet as well as numbness in hands. (Tseng et al., 2010) Though ergot may have unwanted side effects or adverse effects as observed above, however it is also a well-known fact that ergot possess potential advantages as discussed below.

Bromocriptine as treatment of AML: acute amyloid leukemia is a type of cancer that affect the blood and bone marrow. Bromocriptine is a hydrogenated ergot derivatives with dopamine agonist activity, a novel therapy as Anti-AML, induce apoptosis for AML cells. (Lara-Castillo et al., 2016).

Bromocriptine as a treatment of breast cancer: there is a role of dopamine in development of cancer whereas bromocriptine via down regulation of prolactin, result in decrease and suppress the proliferation of MCT-7 cells and induce apoptosis. It also shows effectiveness in idiopathic granulomatous mastitis (IGM) associated with hyperprolactinemia. (Majid et al., 2015).

Ergot still the best as compared to triptans: ergot is still a drug of choice in migraine. It has more good activity as compared to triptans as a powerful vasoconstrictor because it works longer and better for prolong attacks than some triptans. Ergotamine is still very useful and is used in many countries to relief migraine headache. Rectal ergotamine reported higher efficacy as compared to triptans with rectal ergotamine activity of (73\%), while triptan rectally was (63\%). (Marcelo et al., 2003).

Reduce postpartum hemorrhage: Ergotamine IM/IV, as prophylactic agent, shows a good reduction in postpartum hemorrhage when coadminstered with analgesic to reduce the pain. An optimum result predicted with intramuscular injection of ergotamine oxytocin combination called (Syntometrine) at the end of second stage of labor. (Liabsuetrakul, 2007).

Ergot toxicity is due to cytochrome P-450: Ergotamine is metabolized via CYP enzyme and interaction with enzyme inhibitors drugs can increase its level in the body which will lead to toxicity such as in the case of antiviral and antibacterial drugs. Grapefruit also has been reported to increase the level of ergotamine, thus it shows that toxicity as resulted may not be due to ergotamine itself, but due to interaction with drugs inhibiting the enzymes. (Badwin and Ceraldi, 2003) Current review consist of clinical cases reported due to use of ergot. It was observed that the misuse or use without proper medical guidance may result a condition known as ergotism. The major symptoms for ergotism includes; vasospasm, arterial embolism, pain and coldness in feet especially the left one, respiratory depression particularly in neonates, paresthesia and decreased walking distance. The treatment applied for these conditions mostly included vasodilators and Heparin and almost all of the cases observed were due to ergotamine overdose or drug-drug interaction with protease inhibitors. In few of the cases an interaction among ergot alkaloids and other drugs, especially antivirals and azithromycin, was observed when they are administered together. Furthermore, in most of the cases it affected elder people i.e. age of 30 or likewise, however it can affect infants when it's given by medical mistake. Regarding gender it effects both female and male equally however it was observed that patients with HIV disease are more prone to ergotism due to severe interaction. Two cases were observed where toxicity of ergot resulted due to overdose of ergotamine.

Plants remains the well-known sources of treating many ailments mostly working as antioxidant, antidiabetic and antiglycation, (Ahmad et al., 2013 \&t 2014) and the people mostly possesses sound knowledge regarding the use and treatment of most of the plants (Ahmad et al., 2017).

However, in most of the cases the poisoning or toxicity cases are not known to common peoples. Herein, we mentioned ten poisoning cases for ergot where the general and common symptoms observed in elder patients, due to toxicity were; painful and coldness lower extremity especially the left foot, ischemia and purple legs whereas, the symptoms of affected infants were; respiratory depression and pinkish skin. In some cases the lab tests showed normal hemoglobin, white blood cells with a normal temperature (37-37.7C) measured orally. The common treatment used in such toxicity cases was; aspirin, low molecular heparin given IV, and nifedipine orally.

\section{CONCLUSION}

Finally, ergot plant has medicinal uses as well as toxic effects. Current review has found poisoning cases which were mostly due to overdose, mistakenly administered or interaction with anti-HIV drugs for ergot alkaloids. Any disease condition using ergot with proper dose, time and under sound medical supervision whereby the 
interaction with other drugs is taken under consideration, may result a proper therapeutic outcome for ergot.

\section{REFERENCES}

Adam G, Kurt T, Çınar C, Sarıyıldırım A, Resorlu M, Uysal F, Yener AU, Ozcan S. (2014). Ergotamine-induced vasospastic ischemia mimicking arterial embolism. Ulus Travma Acil Cerrahi Derg. 20(4): 291-294.

Ahmad A, Upadhyay A, Ahmad M, Pieters L. (2013). Antioxidant, antliglycation and antimicrobial activities of Ziziphus oxyphylla and Cedrela serrata Extracts. Eur J med plants. 3(4): 520-529.

Ahmad R, Ahmad M, Jahan N, Mehjabeen. (2014). Phytochemical screening and anti-oxidant activity of the two plants Ziziphus oxyphylla Edgew and Cedrela Serrata Royle. Pak J Pharm Sci. 27(5):1477-82.

Ahmad R, Naqvi AA, Ahmad N, Baraka M, Mastour M, Al Sharedah S, Al Ghamdi S, Al Rabae G, Al Ghamdi MS. (2017). Awareness, perception, attitude, and knowledge regarding complementary and alternative medicines (cams) among the pharmacy and medical students of a public university in Saudi Arabia. Arch Pharm Prac. 8(2):51-63.

Baldwin ZK and Ceraldi CC. (2003). Ergotism associated with HIV antiviral protease inhibitor therapy. Journal of Vascular surgery. 37(3): 676-678.

Bangh SA, Hughes KA, Roberts DJ, Kovarik SM. (2005). Neonatal Ergot Poisoning: A Persistent Iatrogenic Illness. American Journal of Perinatology. 22(5): 239-243.

Belser-Ehrlich S, Harper A, Hussey J, Hallock R. (2013). Human and cattle ergotism since 1900: symptoms, outbreaks, and regulations. Toxicol Ind Health. 29(4): 307-16.

Cagatay A, Guler O, Guven K. (2009). Ergotism Caused by Concurrent Use of Ritonavir and Ergot Alkaloids: A Case. Acta chirurgica Belgica. 109(5): 639-640.

Curry JC and Pepine JR. (1977). Effects of Ergonovine in Patients. Circulation journal. 56(5): 803-809.

Demir S, Akın S, Tercan F, Arıboğan A, Oğuzkurt L. (2010). Ergotamine-induced lower extremity arterial vasospasm presenting. Interventional radiology, Turkish Society of Radiology. 16(2): 165-167.

Dhlof C, Maassen A, Brink VD. (2012). Dihydroergotamine, Ergotamine, Methysergde, and sumatriptn-Basic science in Relation to Migraine treatment. The journal of head and face pain banner. 52(4). 707-714.

Eadie MJ. (2003). Convulsive ergotism: epidemics of the serotonin syndrome?. The lancet neurology. 2(7): 429-434.

Eduardo J, Ayarragaray F, Panno M. (2016). Ergotism and Retroviral Therapies: An Association not so Unusual. JSM Foot And Ankle. 1(3): 1-2.

Floss GH. (1976). Biosynthesis of ergot alkaloids and related compounds. Tetrahedran. 32.873-912.

Fröhlich G, Kaplan G, Amann-Vesti B. (2010). Holy fire in an HIV-positive man: a case of 21st-century ergotism. CMAJ. 182(4): 378-380.
Gaye-Saavedra GA, et al., (2016). Transient ischemic attack caused by cerebral ergotism. Arquivos de Neuro-Psiquiatria. 1603(1): 114-114.

Gerhards N, Neubauer L, Tudzynski P, Shu-Ming Li. (2014). Biosynthetic Pathways of Ergot Alkaloids. Toxins. 6(12), 32813295.

Ghali R, Léan JD, Douville Y, Noël HP. (1993). Raymond Labbé. Erythromycin-associated ergotamine. Annals of Vascular surgery. 7(3): 291-296.

Lara-Castillo MC, Cornet-Masana JM, Etxabe A et al., (2016). Repositioning of bromocriptine for treatment of acute myeloid leukemia. Journal of Translational Medicine. 14(1): 1-11.

Lee MR. (2009). The history of ergot of rye (Claviceps purpurea). Journal of Royal College of Physicians of Edinburgh. 39(2). 79-84.

Liabsuetrakul T, Choobun T, Peeyananjarassri K, Islam QM. (2007). Prophylactic use of ergot alkaloids in the third stage of labour. Cochrane Database Syst Rev. 18;(2).

Majid P, Ghasem A, Seyed HH, Abdolkhalegh D. (2015). New Perspective Therapy of Breast Cancer Based on Selective Dopamine Receptor D2 Agonist and Antagonist Effects on MCF-7 Cell Line. Bentham Science Publishers. 10(2): 214-223.

Marcelo E, Stewart JB, Tepper. (2003). Ergotamine and Dihydroergotamine. The New England Center for Headache Stanford USA. 7(1): 55-62.

Mulac D, Humpf HU. (2011). Cytotoxicity and accumulation of ergot alkaloids in human primary cells. Toxicology. 11;282(3): $112-21$.

Mulac D, Lepski S, Ebert F, Schwerdtle T, Humpf HU. (2013). Cytotoxicity and fluorescence visualization of ergot alkaloids in human cell lines. J Agric Food Chem. 16;61(2): 462-71.

Mulac D, Lepski S, Ebert F, Schwerdtle T, Humpf HU. (2013). Cytotoxicity and Fluorescence Visualization of Ergot Alkaloids in Human Cell Lines. Journal of agricultural and food chemistry. 61(2). 462-471.

Musikatavorn K and Suteparak S. (2009). Ergotism unresponsive to multiple therapeutic modulities, including sodium nitroprussside resulting in limb loss. Clinical toxicology. 46(2): 157-158.

Panaccione DG, Ryan KL, Schardl CL, Florea S. (2012) Analysis and modification of ergot alkaloid profiles in fungi. Methods in Enzymology, 515: 267-290.

Schardl CL. (2015). Introduction to the Toxins Special Issue on Ergot Alkaloids. Toxins. 7(10). 4232-4237.

Shimony A, Romem A, Horowitz S, Boehm R, Horowitz J. (2006). Acute coronary syndrome associated with myocardial bridging due to ergotamine treatment for migraine. International journal of cardiology. 113(1): E7-E8.

Sullivan R, Nelsen J, Duggineni S, Holland M. (2012). Management of methylergonovine induced respiratory. Informa healthcare Clinical Toxicology. 51(1): 47-49.

Tseng CW et al., (2010). Acute paresthesia in a patient with migraine. Journal of Clinical Neuroscience. 1328(1): 20-20. 